\title{
Management of posterior cruciate ligament avulsion by cannulated screw fixation
}

\author{
Lamichhane A, Mahara D P \\ Department of Orthopaedics, TU Teaching hospital, Kathmandu, Nepal
}

Correspondence address: Dr Arjun Lamichhane, Department of Orthopaedics, IOM TU Teaching, Hospital, Kathamndu

Email: drajun@gmail.com

\begin{abstract}
Introduction: Posterior cruciate ligament (PCL) is the main posterior stabilizer of the knee. The PCL also plays a role as a central axis controlling and imparting rotational stability to the knee. The most effective management of posterior cruciate ligament (PCL) injuries remains unclear and the natural history of untreated PCL injuries is controversial. The need for surgical repair of displaced bony PCL avulsions is less controversial. Here we present repair of PCL avulsion by screw fixation.
\end{abstract}

Methods: There were total of 18 patients (Male 12 and Female 6) operated for isolated PCL avulsion from tibia during the period from 2002 to 2010. Preoperative evaluation was done clinically and radiologically 'MRI was advised for suspected other ligaments and meniscal injuries. Patients with other ligaments and meniscal injury were not included in this series. All cases operated by simplified approach described by Burks and Schaffer. Avulsed fragment was reduced and fixed with $4 \mathrm{~mm}$ a cannulated screw. Evaluation of the patients were done clinically, radilologically and using functional scale of Tegner-Lysholm.

Results: Out of 18 patients, 12 patients were male and 6 patients were female. Average age of the patients was 29yrs (range 18-47 yrs). Average period of follow up was 31 months (ranging 12 $52 \mathrm{~m}$ ). Range of movement was found full in all patients at the last follow up. Grade 1-2 laxity was found in 8 patients but subjectively they were not aware of the laxity. Average Lysholm score was 91 (range87-97).

Conclusion: Improvised approach by burk and Schaffer is safer and easier than the classical one. Open reduction and fixation with cannulated screw is one of the excellent techniques for tibial avulsion of posterior cruciate ligament.

Key Words: Posterior Cruciate Ligament Avulsion, Stability of knee, Surgical repair, Lysholm score

\section{Introduction}

Posterior cruciate ligament (PCL) is the main posterior stabilizer of the knee. The PCL also plays a role as a central axis controlling and imparting rotational stability to the knee. This injury has received little attention in the past, compared with the ACL; however, the emphasis on the ACL has stimulated increased interest in the treatment of PCL injuries. Isolated injury of the PCL is less common than Anterior Cruciate Ligament (ACL). The most effective management of posterior cruciate ligament (PCL) injuries remains unclear and the natural history of untreated PCL injuries is controversial. Various PCL avulsion surgery techniques have been reported, including alternatives to avoid potentially injurious exposure of the popliteal fossa. The need for surgical repair of displaced bony PCL avulsions is less controversial and several open and arthroscopic techniques have been described ${ }^{1}$ Here we present Posterior cruciate ligament avulsion surgically fixed with cannulated screw.

\section{Methods}

There were total of 18 patients (Male 12 and Female 6) operated for isolated PCL avulsion from tibia during the 
period from 2002 to 2010 . Average age of the patients was 29yrs (range 18-47 yrs) (Chart 1). Preoperative evaluation was done clinically and radiologically ( Fig.1a). MRI was advised for suspected other ligaments and meniscal injuries. Patients with other ligaments and meniscal injury were not included in this series.

\section{Operative Technique}

Tourniquet is applied high up in the thigh. The patient is positioned in the prone position and the lower extremity is held in 30 degree flexion at the knee joint over a bolster at ankle. Simplified approach described by Burks and Schaffer ${ }^{2}$ consist of An inverted L incision is made over the posteromedial corner of the knee joint: The skin incision is a gentle curve with a horizontal end near the flexion crease of the knee and a vertical limb overlying the medial aspect of the gastrocnemius muscle. Dissection is carried to the deep fascial layer, which is incised vertically over the medial head of the gastrocnemius. T'he medial border of the medial gastrocnemius is identified, and the interval is developed between it and the semimembranosus tendon. This plane is developed by blunt dissection until the posterior joint capsule is reached. Medial head of gastrocnemius is retracted laterally so neurovascular structures are away from surgical field (Fig. 1b).

At this point a vertical incision is made through the posterior capsule. The contents of the posterior intercondylar notch and the tibial attachment of the PCL are exposed. Avulsed fragment was isolated; the crater was cleaned, reduced the fragment to the site and fixed with $4 \mathrm{~mm}$ cannulated screw (Fig. 1c). Stability was checked with range of movement of the knee. Posterior capsule was closed, and the wound was closed with suction drain.

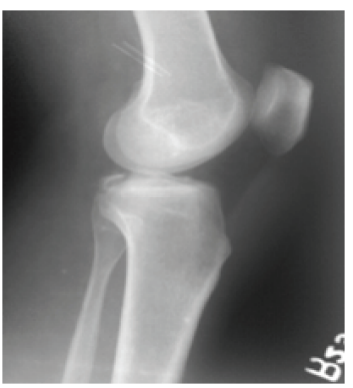

(a)

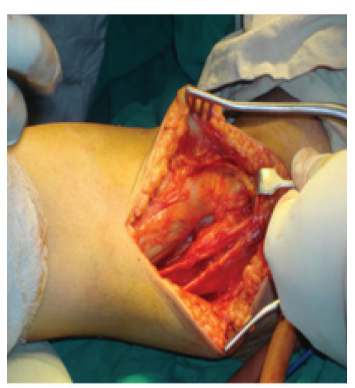

(b)

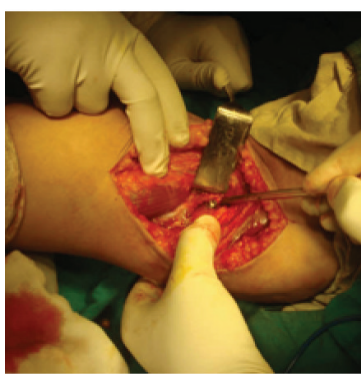

(c)

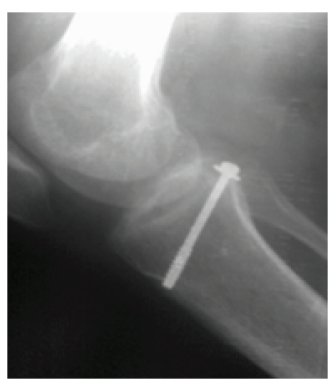

(d)
Fig.1: Illustration of (a) baseline x-ray of the knee, (b) posteromedial approach by Burks and Schaffer approach, (c) fixation with cannulated screw, and (d) after union of the injury.

The limb was immobilized in extension. The operated limb was immobilized for 4 weeks and mobilized with weight bearing crutch walking. Physiotherapy was started after removal of the slab,that included range of motion, mobility, and quadriceps strengthening. Clinical examination was performed, radiographs were obtained ( Fig. 1d) and functional tests of Lysholm score was obtained. Return to heavy labor or competitive sports activities were restricted until after 6 to 9 months when sufficient strength, range of motion, and proprioceptive skills had returned. Evaluation of the patients were done clinically, radilologically and using functional scale of Tegner-Lisholm.

Grading the Tegner Lysholm Knee Scoring Scale was done as:

- $<65$ Poor

- 65-83Fair

- 84-90Good

- >90Excellent

\section{Results}

Table 1: Descriptive data: sex, age , posterior drawer test, wasting, involved knee and Tegner - Lysholm score.

$\begin{array}{lllllll}\text { Case no } & \text { Sex } & \text { Age } & \begin{array}{l}\text { PDT } \\ \text { grade }\end{array} & \begin{array}{l}\text { Thigh } \\ \text { wasting } \\ \mathrm{cm} .\end{array} & \text { Knee } & \begin{array}{l}\text { Lysholm } \\ \text { score }\end{array} \\ 1 & \text { M } & 23 & 1 & .5 \mathrm{~cm} & \mathrm{~L} & 89 \\ 2 & \mathrm{M} & 28 & 0 & - & \mathrm{R} & 95 \\ 3 & \mathrm{~F} & 21 & 0 & - & \mathrm{R} & 94 \\ 4 & \mathrm{M} & 46 & 2 & .5 & \mathrm{R} & 88\end{array}$




$\begin{array}{lllllll}5 & \text { F } & 47 & 1 & 1 & \mathrm{~L} & 87 \\ 6 & \mathrm{M} & 19 & 0 & - & \mathrm{R} & 93 \\ 7 & \mathrm{M} & 25 & 0 & - & \mathrm{L} & 92 \\ 8 & \mathrm{~F} & 26 & 1 & .5 & \mathrm{R} & 89 \\ 9 & \mathrm{M} & 22 & 1 & .5 & \mathrm{~L} & 91 \\ 10 & \mathrm{M} & 43 & 0 & - & \mathrm{R} & 91 \\ 11 & \mathrm{~F} & 33 & 1 & - & \mathrm{L} & 90 \\ 12 & \mathrm{M} & 24 & 0 & - & \mathrm{L} & 93 \\ 13 & \mathrm{M} & 29 & 0 & .5 & \mathrm{R} & 91 \\ 14 & \mathrm{M} & 21 & 1 & - & \mathrm{R} & 92 \\ 15 & \mathrm{~F} & 19 & 0 & - & \mathrm{R} & 91 \\ 16 & \mathrm{M} & 31 & 0 & .5 & \mathrm{R} & 91 \\ 17 & \mathrm{~F} & 26 & 1 & .5 & \mathrm{~L} & 89 \\ 18 & \mathrm{M} & 29 & 0 & - & \mathrm{R} & 92\end{array}$

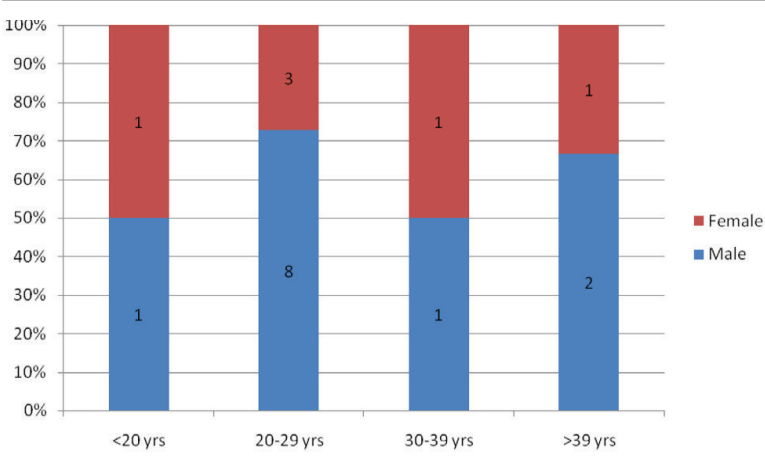

Fig. 2: Age and sex distribution

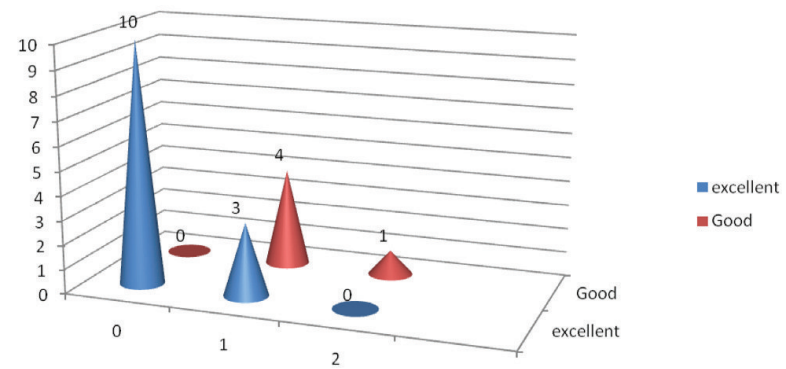

Fig. 3: Posterior drawer test grade and Lysholm score grading

The average interval of injury and the surgery was 12 days (Range 7 dyas -2 months). Road traffic accident as mode of injury was found in 13 cases and fall / slip injury in 5 cases. Union of the avulsed fragment occurred in all cases by 6 weeks. Average period of follow up was 31 moths (ranging 12 - $52 \mathrm{~m}$ ). Range of movement was found full in the last follow up. Grade 1-2 laxity was found in 8 patients but subjectively they were not aware of the laxity had excellent lysholm score in some patients( Fig 3).

Mild wasting (range $.5-1 \mathrm{~cm}$ ) of the thigh muscle was found in 8 cases but the strength of the quadriceps muscle was as strong as contra lateral side. Average tegner Lysholm score was 91(Chart 1).

\section{Discussion}

The past decade has seen a renewed interest in the posterior cruciate ligament (PCL) as numerous studies have reported on its anatomy, biomechanics, and various reconstruction techniques. Despite this, our knowledge of the PCL still lags behind that of other knee ligamentous structures, particularly the anterior cruciate ligament (ACL). The more infrequent nature of PCL injury, lack of familiarity with its injury and treatment, increased surgical risks, and poor results of early surgical treatment are all factors that have led to this disparity in knowledge.

However, with advances in basic science knowledge pertaining to the PCL and improved awareness and imaging modalities, PCL injuries are being more accurately diagnosed and treated. This has led to more focus on the various treatment options and their results.

The incidence of PCL tears is reported to be between $1 \%$ and $44 \%$ of acute knee injuries ${ }^{4}$. This includes midsubstance tear from either end and bony avulsion from tibia. Common mechanism of injury of the $\mathrm{PCl}$ injury is Dashboard injury, but all cases in this series had history of RTA and the mechanism as stated seems complex one. Direct posterior approach (Abbott and Carpenter)for the avulsion of PCL was the commonly used approach in many series. Burk and Schaffer approach now is safe and easy ${ }^{2}$. In literature, surgical indications for PCL injuries treatment remain controversial, but in PCL avulsion, surgical reinsertion of the avulsioned fragment is a recommended procedure ${ }^{5,6}$. Posterior cruciate ligament rupture leads to an increase in the passive sagittal laxity of the medial compartment of the knee so alters the kinematics of the medial compartment of the knee, resulting in "fixed" anterior subluxation of the medial femoral condyle (posterior subluxation of the medial tibial plateau $)^{7}$. This results in development of osteoarthritis in medial compartment in patients with chronic posterior instability, so necessitates the repair or reconstruction of the injured PCL.

Some amount of wasting was present in majority of the cases since they were not on regular follow up for physiotherapy and were not aware of the wasting also.

Clinical outcomes achieved at the objective evaluation suggest that this injury should be interpreted not only as a purely bone injury, but also as a bone-ligament injury ${ }^{8}$. 
Despite Grade 1-2 posterior instability in this series, patients were satisfied since the end point of posterior drawer test was bony.

K Donald et al. ${ }^{9}$ in a prospective study of natural history of isolated PCL injury found that there was no direct relationship between the objective finding of grade of PCL laxity and subjective knee function.

Inoue et al. ${ }^{10}$ conducted a prospective study intending to evaluate clinical prognosis of open reduction and PCL avulsion fracture fixation. Their study showed that approximately $60 \%$ of the 31 studied patients showed a certain degree of posterior instability on operated knees when compared to intact knees, even after anatomical reduction and stiff fixation of the bone fragment.

Piedade SR and Mischan $\mathrm{MM}^{11}$ in 21 case series, found good in $43 \%$ and excellent in $57 \%$ in a subjective evaluation( Lysholm) and residual posteriorisation of $+(.5 \mathrm{~cm})$ in $57 \%$ and $++(1 \mathrm{~cm})$ in $38 \%$ of the cases in posterior drawer test. So, stated that surgical treatment of avulsion fracture of the posterior cruciate ligament provides satisfactory subjective outcomes despite objective laxity.

Boynton and Tietjens ${ }^{12}$ reviewed 38 patients with isolated rupture of the PCL in a long-term study showing that the majority of patients were symptomatic, with radiographic evidence of osteoarthritis increasing with time from injury. Therefore, it would appear that conservative management of PCL ruptures produces good results in the short and medium term, but in the longer term, patients develop pain due to articular damage. This study also justifies the surgical fixation of PCL avulsion.

\section{Conclusion}

Improvised approach by burk and Schaffer is safer and easier than the classical one. Open reduction and fixation with cannulated screw is one of the excellent techniques for tibial avulsion of posterior cruciate ligament. Patient satisfaction was not dependent upon some amount of wasting and laxity but with excellent Lysholm score

\section{References}

1. Nicandri GT, Klineberg EO, Wahl CJ, Mills WJ. Treatment of posterior cruciate ligament tibial tibial avulsion fractures through a modified open posterior approach: operative technique and 12-48 month outcomes. Journal of Orthopaedic Trauma 2008; 22(5): 317-324

2. Robertt T. Burks, John J . Schaffer . A Simplified Approach to the Tibial Attachment of the Posterior Cruciate Ligament: Clin Orthop Relat Res 1990;
(254):216-19

3. Tegner Y, Lysholm J. Rating systems in the evaluation of knee ligament injuries. Clin Orthop Relat Res 1985; (198):43-9.

4. K.Donald Shelbourne, Thorp J. Davis, and Dipak V. Patel The Natural History of Acute, Isolated, Nonoperatively Treated Posterior Cruciate Ligament Injuries: A Prospective Study Am J Sports Med May 1999; 27: 276-283.

5. William M. Wind, Jr, John A. Bergfeld, and Richard D. Parker Clinical Sports Medicine Update: Evaluation and Treatment of Posterior Cruciate Ligament Injuries: Revisited Am J Sports Med October 2004; 32 1765-1775;

6. David J. Deehan M.D., Leo A. Pinczewski F.R.A.C.S. Arthroscopic reattachment of an avulsion fracture of the tibial insertion of the posterior cruciate ligament. Arthroscopy: The Journal of Arthroscopic \& Related Surgery 2001;17(4):422-425.

7. M Logan, A Williams, J Lavelle, W Gedroyc, M Freeman. The Effect of Posterior Cruciate Ligament Deficiency on Knee Kinematics: Am. J. Sports Med. 2004; 32(8):1915-1922.

8. S R Piedade, M M Mischan. Surgical treatment of avulsion of the knee PCL tibial insertion: experience with 21 cases: Acta Orthop Bras. 2007; 15(5): 272275.

9. K. Donald Shelbourne, $\dagger$ MD, Thorp J. Davis, MD, and Dipak V. Patel, MD. The Natural History of Acute, Isolated, Nonoperatively Treated Posterior Cruciate Ligament Injuries Am J Sports Med 1999; Vol. 27, No. 3

10. Inoue M, Yasuda K, Kondo E, Saito K, Ishibe M. Primary repair of posterior cruciate ligament avulsion fracture. The effect of occult injury in the midsubstance on postoperative instability. Am J Sports Med. 2004; 32:1230-7.

11. Piedade SR, Mischan MM. Surgical treatment of avulsion fractures of the knee PCL tibial insertion: experience with 21 cases. Acta Ortop Bras. [serial on the Internet]. 2007; 15(5): 272-275.

12. Boynton MD, Tietjens BR. Long-term followup of the untreated isolated posterior cruciate ligamentdeficient knee. Am J Sports Med. 1996; 24:306-310. 